\title{
Qing Hua Chang Yin inhibits the LPS-induced activation of the IL-6/STAT3 signaling pathway in human intestinal Caco-2 cells
}

\author{
XIAO KE ${ }^{1}$, GUANGHONG HU $^{1}$, WENYI FANG ${ }^{1}$, JINTUAN CHEN $^{1}$, XIN ZHANG ${ }^{1}$,

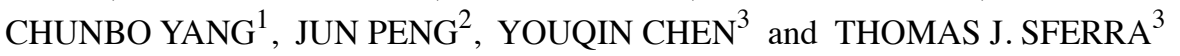 \\ ${ }^{1}$ Department of Gastroenterology, The Second Affiliated Hospital of Fujian University of Traditional Chinese Medicine, \\ Fuzhou, Fujian 350003; ${ }^{2}$ Academy of Integrative Medicine, Fujian University of Traditional Chinese Medicine, \\ Minhou Shangjie, Fuzhou, Fujian 350122, P.R. China; ${ }^{3}$ Rainbow Babies and Children's Hospital, \\ Case Western Reserve University School of Medicine, Cleveland, OH 44106, USA
}

Received November 22, 2014; Accepted January 21, 2015

DOI: 10.3892/ijmm.2015.2083

\begin{abstract}
Increasing evidence indicates that the pathogenesis of ulcerative colitis (UC) is highly regulated by the interleukin-6 (IL-6)/signal transducer and activator of transcription 3 (STAT3) pathway and its negative feedback regulator, suppressor of cytokine signaling 3 (SOCS3). Therefore, modulating the signaling feedback loop of IL-6/STAT3/SOCS3 may prove to be a novel therapeutic approach for the treatment of UC. Qing Hua Chang Yin (QHCY) is a traditional Chinese formulation that has long been used in clinic for the treatment of UC. We have previously reported that QHCY ameliorates acute intestinal inflammation in vivo and in vitro through the suppression of the nuclear factor- $\mathrm{\kappa B}(\mathrm{NF}-\mathrm{\kappa B})$ pathway. In the present study, in order to further elucidate the mechanisms responsible for the anti-inflammatory activities of QHCY, we stimulated human intestinal Caco-2 cells with lipopolysaccharide (LPS) to create an in vitro model of an inflamed human intestinal epithelium, and evaluated the effects of QHCY on the IL-6/STAT3/SOCS3 signaling network in inflamed Caco-2 cells. The levels of IL-6 were measured by ELISA and the levels of STAT3 and SOCS3 were measured by western blot analysis. We found that QHCY significantly inhibited the LPS-induced secretion of pro-inflammatory IL-6 in the Caco-2 cells in a dose-dependent manner. Moreover, $\mathrm{QHCY}$ profoundly
\end{abstract}

Correspondence to: Dr Xiao Ke, Department of Gastroenterology, The Second Affiliated Hospital of Fujian University of Traditional Chinese Medicine, 13 Hu Dong Zhi Road, Fuzhou, Fujian 350003, P.R. China

E-mail:drkxkx@163.com

Abbreviations: QHCY, Qing Hua Chang Yin; TCM, traditional Chinese medicine; UC, ulcerative colitis; IBD, inflammatory bowel disease; IL-6, interleukin-6; STAT3, signal transducer and activator of transcription 3; LPS, lipopolysaccharide

Key words: Qing Hua Chang Yin, traditional Chinese medicine, ulcerative colitis, interleukin-6/signal transducer and activator of transcription 3 pathway suppressed the LPS-induced phosphorylation of Janus-activated kinase 1 (JAK1), JAK2 and STAT3. Furthermore, treatment with QHCY markedly augmented the expression of SOCS3. Taken together, the findings of the present study suggest that the modulation of the IL-6/STAT3/SOCS3 signaling network may be one of the mechanisms through which QHCY exerts its anti-inflammatory effects.

\section{Introduction}

Inflammatory bowel disease (IBD), including Crohn's disease (CD) and ulcerative colitis (UC), constitutes one of the most prevalent gastrointestinal disease burdens worldwide. Patients with IBD may present with any of the following predominant symptoms: severe abdominal pain, vomiting, diarrhea, rectal bleeding and weight loss. More importantly, IBD is associated with an increased risk of developing colorectal cancer (1-5). Present treatment strategies for IBD are directed at eliminating inflammation in order to relieve symptoms and heal the intestinal mucosa. However, the majority of the currently used drugs attenuate the symptoms of the disease rather than effectively maintaining disease remission. In addition, significant adverse effects prohibit their long-term use (6-8). Thus, there is a need for the development of additional safe and effective therapies for these patients. Recently, complementary therapies, such as natural products, dietary components and traditional Chinese medicine (TCM), have become attractive approaches for the treatment of IBD (9-12).

Considerable evidence has implicated the involvement of pro-inflammatory cytokines, particularly interleukin-6 (IL-6), in the pathogenesis of IBD $(13,14)$. Several studies have reported that substantially elevated IL-6 serum levels in patients with IBD (14-16). Moreover, studies have demonstrated a significant positive correlation between the levels of IL-6 and disease severity (17-23). IL-6 preferentially activates signal transducer and activator of transcription 3 (STAT3) proteins, and their phosphorylation is mediated through the activation of Janus-activated kinases (JAK). Phosphorylated STAT3 forms homodimers and then translocates into the nucleus to regulate the transcription of pro-inflammatory 
genes, such as cytokines and chemokines $(24,25)$. One of the target genes of the IL-6/STAT3 pathway is the suppressor of cytokine signaling (SOCS)3. SOCS comprises a family of proteins that regulates negative feedback to the JAK/STAT cytokine signaling cascade $(26,27)$. Both human observations and experimental evidence from mouse studies have indicated that the IL-6/STAT3/SOCS3 pathway plays a key role in regulating intestinal epithelial cell homoeostasis, and an imbalance between SOCS3 expression and IL-6/STAT3 signaling leads to inflammation and eventually to inflammation-induced carcinogenesis (28-31). Thus, modulating the signaling feedback loop of IL-6/STAT3/SOCS3 may prove to be a novel therapeutic approach for the treatment of IBD.

Natural products, including those used in TCM, manifest potential anti-inflammatory activity. Qing Hua Chang Yin (QHCY) is a TCM formulation that consists of a combination of 11 herbs, namely Herba et Gemma Agrimoniae, Coptis chinensis Franch., Radix Sanguisorba officinalis, Radix Paeoniae Rubra, Elettaria cardamomum, Magnolia officinalis, Artemisia capillaris Thunb., Eupatorium fortunei, Semen Coicis, Semen Dolichoris Album and Poria cocos. Collectively, these components confer QHCY with the properties of the clearing of heat and dampness, as well as strengthening the spleen to nourish vitality (tonifying the Spleen Qi in Chinese). QHCY has long been used in China to clinically treat UC (32-37). We recently reported that QHCY ameliorates acute intestinal inflammation in vivo and in vitro through the suppression of the nuclear factor (NF) $\kappa$ B pathway $(38,39)$. In this study, to further elucidate the mechansims responsible for the anti-inflammatory activities of QHCY, we stimulated Caco-2 cells to create an in vitro model of an inflamed human intestinal epithelium, and evaluated the effects of QHCY on the IL-6/STAT3/SOCS3 signaling network in inflamed Caco-2 cells.

\section{Materials and methods}

Materials and reagents. Dulbecco's modified Eagle's medium (DMEM), fetal bovine serum (FBS), penicillin-streptomycin and trypsin-ethylenediaminetetraacetic acid (EDTA) were purchased from Life Technologies (Carlsbad, CA, USA). Lipopolysaccharide (LPS) from Escherichia coli serotype 055:B5 was purchased from Sigma-Aldrich (St. Louis, MO, USA). Antibodies for western blot analysis were obtained from Cell Signaling Technology, Inc. (Beverly, MA, USA). The human IL-6 enzyme-linked immunosorbent assay (ELISA) kit was obtained from BD Pharmingen (San Diego, CA, USA). All other reagents, unless otherwise stated, were obtained from Sigma-Aldrich.

Preparation of $Q H C Y$. QHCY was prepared as described in our previous study (39). A total of $220 \mathrm{~g}$ dehydrated Herba et Gemma Agrimoniae, 33 g Coptis chinensis Franch., 100 g Radix Sanguisorba officinalis, 110 g Radix Paeoniae Rubra, 56 g Elettaria cardamomum, 110 g Magnolia officinalis, 110 g Artemisia capillaris Thunb., 110 g Eupatorium fortunei, $220 \mathrm{~g}$ Semen Coicis, $110 \mathrm{~g}$ Semen Dolichoris Album and 220 g Poria cocos were extracted with boiling water 3 times. The extracts were then combined and concentrated by boiling to a final volume of $1,000 \mathrm{ml}$. The final concentration of the QHCY crude drug was $\sim 1.4 \mathrm{mg} / \mathrm{ml}$.
Cell culture. Cell cultrure was carried out as described in a previous study of ours (39). Human colon cancer Caco-2 cells were purchased from the American Type Culture Collection (ATCC; Rockville, MA, USA). Cells (from passages 20-40) were grown in DMEM containing $10 \%$ (v/v) FBS, $1,000 \mathrm{mg} / \mathrm{l}$ of glucose, $50 \mathrm{U} / \mathrm{ml}$ penicillin and $50 \mu \mathrm{g} / \mathrm{ml}$ streptomycin in a $37^{\circ} \mathrm{C}$ humidified incubator with $5 \% \mathrm{CO}_{2}$. Cells were subcultured at $85-90 \%$ confluence. The Caco- 2 cells usually reached confluence 3 days after seeding and differentiated into enterocyte-like cells 18-20 days post-confluence. Fully differentiated cells were used for the experiments. On the day of the experiment, the medium was removed and the cells were washed twice with DMEM supplemented with $0.5 \%$ FBS.

ELISA. As previously described (39), differentiated Caco-2 cells (20 days post-confluence) in 24-well plates were incubated with various concentrations of QHCY for $1 \mathrm{~h}$ prior to stimulation with LPS $(1 \mu \mathrm{g} / \mathrm{ml})$ for $24 \mathrm{~h}$. At the end of the experiment, the supernatants were collected by centrifugation of the cell culture medium at 3,000 $\mathrm{xg}$ for $10 \mathrm{~min}$. The secretion levels of IL-6 were measured using a human IL-6 ELISA kit according to the manufacturer's instructions. All samples were assayed in triplicate. The absorbance was read at $450 \mathrm{~nm}$.

Western blot analysis. Differentiated Caco-2 cells (20 days post-confluence) in 6-well plates were incubated with various concentrations of QHCY for $1 \mathrm{~h}$ prior to stimulation with LPS $(1 \mu \mathrm{g} / \mathrm{ml})$ for $30 \mathrm{~min}$ for the detection of phosphoryalted (p-)STAT3 and p-JAK1/2, or $24 \mathrm{~h}$ for the measurement of SOCS3 protein expression. As previously described (39), at the end of the experiment, the cells were washed with ice-cold phosphate-buffered saline (PBS). The cells were then lysed with cell lysis buffer (50 mM Tris- $\mathrm{HCl}, \mathrm{pH} 7.4,150 \mathrm{mM} \mathrm{NaCl}$, $0.5 \%$ NP-40, $5 \mathrm{mM}$ EDTA, $50 \mathrm{mM} \mathrm{NaF}$ and $1 \mathrm{mM}$ PMSF) containing protease and phosphatase inhibitor (PI) cocktails. The cell lysate was centrifuged at $10,000 \mathrm{x} \mathrm{g}$ at $4^{\circ} \mathrm{C}$ for $10 \mathrm{~min}$ and the supernatant was collected. The protein concentration was quantified using BCA. Equivalent amounts of protein were resolved in $12 \%$ sodium dodecyl sulfate-polyacrylamide gel electrophoresis (SDS-PAGE) gels and electroblotted. The PVDF membranes were blocked with 5\% skimmed milk and probed with primary antibodies against STAT3 (\#4904), p-STAT3 (\#9145), JAK1 (\#3332), p-JAK1 (\#4904), JAK2 (\#3230), p-JAK2 (\#3771), SOCS3 (\#2932) or $\beta$-actin (\#4967) (1:1,000; all from Cell Signaling Technology, Inc.) overnight at $4^{\circ} \mathrm{C}$ and then again with appropriate HRP-conjugated secondary antibody followed by enhanced chemiluminescence detection.

Statistical analysis. Data were analyzed using the SPSS package for Windows (version 11.5; SPSS, Inc., Chicago, IL, USA). Statistical analysis of the data was performed with the Student's t-test and one-way ANOVA. A value of $\mathrm{P}<0.05$ was considered to indicate a statistically significant difference.

\section{Results}

QHCY inhibits the LPS-induced release of IL-6 in intestinal epithelial cells. Since the pro-inflammatory cytokine, IL-6, plays an important role in the inflammatory process, we first examined the effects of QHCY on the production of IL-6 


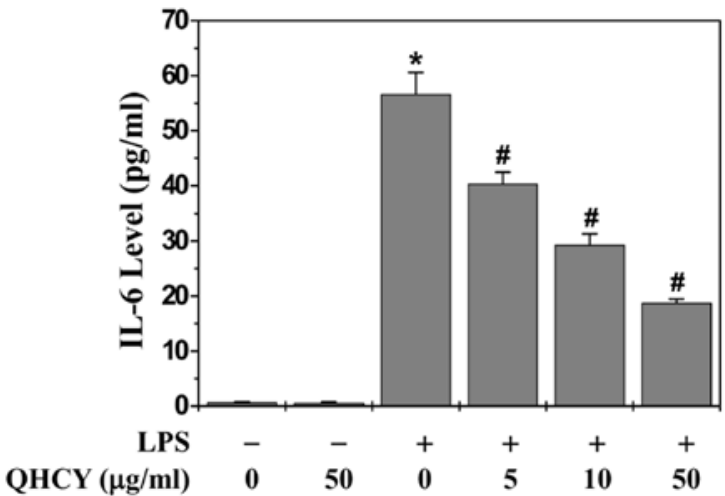

Figure 1. Effect of Qing Hua Chang Yin (QHCY) on the protein secretion levels of interleukin (IL)-6 in lipopolysaccharide (LPS)-stimulated Caco-2 cells. Differentiated Caco-2 cells (20 days post-confluence) in 24-well plates were incubated with the indicated concentrations of $\mathrm{QHCY}$ for $1 \mathrm{~h}$ prior to stimulation with LPS for $24 \mathrm{~h}$. The secretion levels of IL-6 were examined by ELISA. Data are presented as the means \pm SD (error bars) from at least 3 independent experiments. ${ }^{*} \mathrm{P}<0.05$ vs. control cells; ${ }^{*} \mathrm{P}<0.05$ vs. cells treated with LPS but without QHCY.

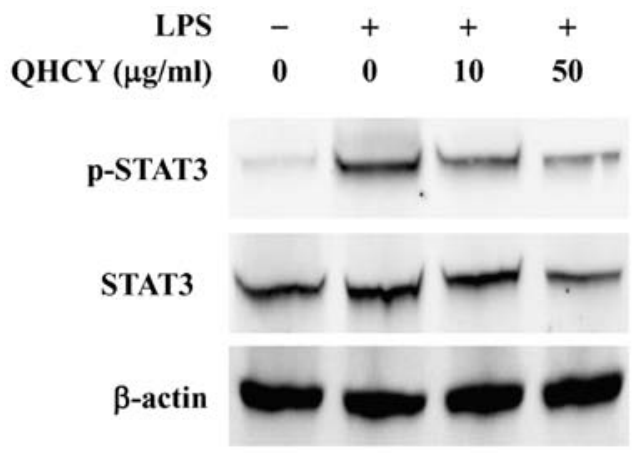

Figure 2. Effect of Qing Hua Chang Yin (QHCY) on lipopolysaccharide (LPS)-induced signal transducer and activator of transcription 3 (STAT3) phosphorylation in Caco-2 cells. Differentiated Caco-2 cells (20 days postconfluence) in 6-well plates were incubated with the indicated concentrations of QHCY for $1 \mathrm{~h}$ prior to stimulation with LPS for $30 \mathrm{~min}$. STAT3 phosphorylation was determined by western blot analysis. $\beta$-actin was used as the internal control. Images are representative of 3 independent experiments.

in differentiated Caco-2 cells stimulated with LPS (Fig. 1). Exposure to LPS led to a significant increase in the production of IL-6, while pre-treatment with QHCY profoundly inhibited the LPS-induced secretion of IL-6 in a dose-dependent manner (Fig. 1).

QHCY inhibits the LPS-induced activation of the JAK/ STAT3 signaling pathway in intestinal epithelial cells. STAT3 has been implicated as a key transcription factor in inflammatory signaling cascades triggered by LPS and other cytokines (40-42). We therefore assessed whether the inhibitory effect of QHCY on the release of IL-6 in differentiated Caco-2 cells stimulated with LPS occurred through the inhibition of STAT signaling. Differentiated Caco-2 cells were pre-treated with or without QHCY at various doses for $1 \mathrm{~h}$, followed by stimulation with LPS $(1 \mu \mathrm{g} / \mathrm{ml})$ for $30 \mathrm{~min}$; total proteins were extracted and analyzed by western blot analysis. The stimulation of the cells with LPS resulted in an

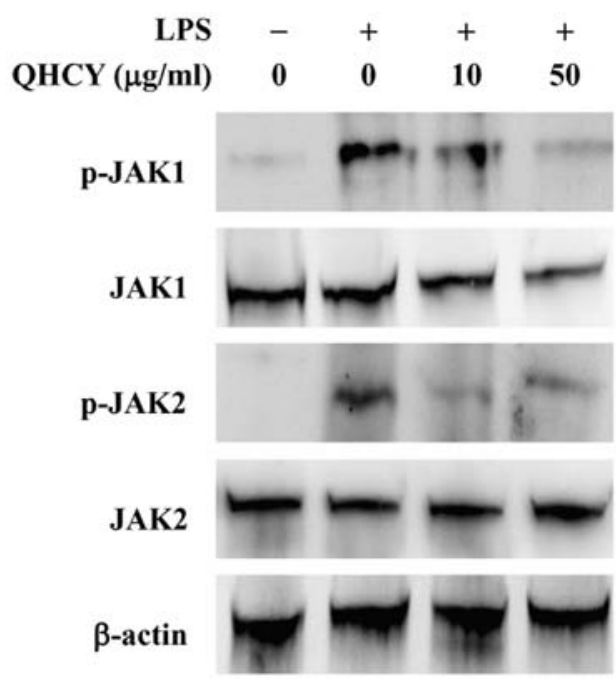

Figure 3. Effect of Qing Hua Chang Yin (QHCY) on the lipopolysaccharide (LPS)-induced Janus-activated kinase (JAK) phosphorylation in Caco-2 cells. Differentiated Caco- 2 cells (20 days post-confluence) in 6-well plates were incubated with the indicated concentrations of QHCY for $1 \mathrm{~h}$ prior to stimulation with LPS for $30 \mathrm{~min}$. JAK phosphorylation was determined by western blot analysis. $\beta$-actin was used as the internal control. Images are representative of 3 independent experiments.

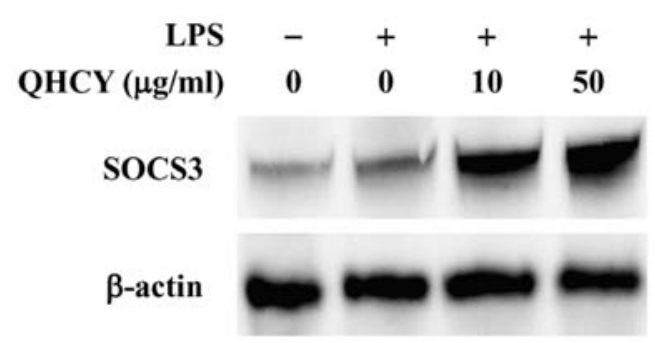

Figure 4. Effect of Qing Hua Chang Yin (QHCY) on the expression of suppressor of cytokine signaling 3 (SOCS3) in Caco-2 cells. Differentiated Caco-2 cells (20 days post-confluence) in 6-well plates were incubated with the indicated concentrations of QHCY for $1 \mathrm{~h}$ prior to stimulation with lipopolysaccharide (LPS) for $24 \mathrm{~h}$. The protein expression levels of SOCS3 were determined by western blot analysis. $\beta$-actin was used as the internal control. Images are representative of 3 independent experiments.

increased STAT3 phosphorylation (Fig. 2). By contrast, the increased phosphorylation of STAT3 was prevented by QHCY in a dose-dependent manner. Since STAT3 has been found to be activated by JAKs in response to LPS $(43,44)$, we then examined the effects of QHCY on the LPS-induced activation of JAK1 and JAK2. Pre-treatment with QHCY attenuated the LPS-induced phosphorylation of JAK1 and JAK2 (Fig. 3).

QHCY increases the expression of SOCS3 in LPS-stimulated intestinal epithelial cells. As a STAT3 transcriptional target, SOCS3 is a key negative regulator of the IL-6 signaling pathway, forming a negative feedback loop to inhibit JAK activation $(26,27,45)$. To further explore the mechanisms responsible for the anti-inflammatory activity of QHCY, we investigated its effects on SOCS3 expression. Minimal SOCS3 induction by LPS was observed compared to the untreated cells (Fig. 4). However, QHCY markedly increased the protein expression of SOCS3, which is consistent with the above 
mentioned observation that QHCY inhibited the LPS-induced activation of the JAK/STAT3 signaling pathway.

\section{Discussion}

UC is a phenotype of IBD characterized by chronic recurrent colonic inflammation. Despite the advancements that have been made in the treatment of patients with current popular medications, a large number of patients does not remain in remission. Thus, the need for new treatment options critical. Natural products, dietary components and TCM are becoming an attractive approach for the treatment of various inflammatory disorders, including IBD. QHCY is a TCM formulation that has been demonstrated to be effective in China for the clinical treatment of UC (32-37). We have previously demonstrated that QHCY exerts an anti-inflammatory effect by acting as a potent inhibitor of NF- $\mathrm{KB}$ activation $(38,39)$. However, the precise mechanisms responsible for its anti-inflammatory activity remain to be further elucidated.

The aim of the present study was to determine whether QHCY exerts its anti-inflammatory effects through the abrogation of the IL-6/STAT3/SOCS3 signaling pathway. Using LPS-stimulated Caco-2 cells as an in vitro inflammatory model of the human intestinal epithelium, we first explored the effects of QHCY on the LPS-induced secretion of IL-6. We observed that pre-treatment with QHCY significantly suppressed the LPS-induced release of IL-6 into the cell culture medium, indicating that QHCY exerts an antiinflammatory effect on LPS-induced inflammatory responses in intestinal epithelial cells. We consistently found that QHCY significantly suppressed the LPS-triggered phosphorylation of STAT3 in a dose-dependent manner, which is similar to the inhibitory effect of QHCY on the release of IL-6 from differentiated Caco-2 cells stimulated with LPS. These findings suggest that QHCY inhibits the LPS-induced production of pro-inflammatory factors, at least in part, by blocking STAT3 activation and subsequent transcription. We also observed that QHCY attenuated the LPS-induced phosphorylation of JAK1 and JAK2 in differentiated Caco-2 cells, which is in accordance with the reduced phosphorylation state of STAT3. This finding indicates that QHCY inhibits the phosphorylation of STAT3, possibly through the inactivation of JAK1 and JAK2 in LPS-induced inflammatory responses. In addition, we found that QHCY increased the expression of SOCS3 in differentiated Caco-2 cells stimulated with LPS.

In conclusion, to the best of our knowledge, the present study provides the first evidence of the QHCY suppression of LPS-induced inflammatory responses in intestinal epithelial cells through the inhibition of the JAK-STAT signaling pathway and the increased expression of SOCS3.

\section{Acknowledgements}

This study was sponsored by the National Natural Science Foundation of China (no. 81173432).

\section{References}

1. Eaden JA, Abrams KR and Mayberry JF: The risk of colorectal cancer in ulcerative colitis: a meta-analysis. Gut 48: 526-535, 2001.
2. Eaden J: Review article: colorectal carcinoma and inflammatory bowel disease. Aliment Pharmacol Ther 20 (Suppl 4): 24-30, 2004.

3. Ekbom A, Helmick C, Zack M and Adami HO: Ulcerative colitis and colorectal cancer. A population-based study. N Engl J Med 323: 1228-1233, 1990.

4. Canavan C, Abrams KR and Mayberry J: Meta-analysis: colorectal and small bowel cancer risk in patients with Crohn's disease. Aliment Pharmacol Ther 23: 1097-1104, 2006.

5. Gillen CD, Walmsley RS, Prior P, et al: Ulcerative colitis and crohn's disease: a comparison of the colorectal cancer risk in extensive colitis. Gut 35: 1590-1592, 1994.

6. Isaacs KL, Lewis JD, Sandborn WJ, et al: State of the art: IBD therapy and clinical trials in IBD. Inflamm Bowel Dis 11: (Suppl 1): S3-S12, 2005.

7. Kaser A and Tilg H: Novel therapeutic targets in the treatmentof IBD. Expert Opin Ther Targets 12: 553-563, 2008.

8. Hanauer SB: Inflammatory bowel disease. N Engl J Med 334: 841-848, 1996.

9. Bensoussan M, Jovenin N, Garcia B, et al: Complementary and alternative medicine use by patients with inflammatory bowel disease: results from a postal survey. Gastroenterol Clin Biol 30: 14-23, 2006.

10. Stickel F and Schuppan D: Herbal medicine in the treatment of liver diseases. Dig Liver Dis 39: 293-304, 2007.

11. Treasure J: Herbal medicine and cancer: an introductory overview. Semin Oncol Nurs 21: 177-183, 2005.

12. Langmead L and Rampton DS: Review article: complementary and alternative therapies for inflammatory bowel disease. Aliment Pharmacol Ther 23: 341-349, 2006.

13. MacDonald TT, Hutchings P, Choy MY, et al: Tumour necrosis factor-alpha and interferon-gamma production measured at the single cell level in normal and inflamed human intestine. Clin Exp Immunol 81: 301-305, 1990.

14. Gross V, Andus T, Caesar I, et al: Evidence for continous stimulation of interleukin-6 production in Crohn's disease. Gastroenterology 102: 514-519, 1992.

15. Holub MC, Makó E, Dévay T, et al: Increased interleukin-6 levels, interleukin- 6 receptor and gp130 expression in peripheral lymphocytes of patients with inflammatory bowel disease. Scand J Gastroenterol Suppl 228: 47-50, 1998.

16. Reinecker HC, Steffen M, Witthoeft T, et al: Enhanced secretion of tumour necrosis factor-alpha, IL-6, and IL-1 beta by isolated lamina propria mononuclear cells from patients with ulcerative colitis and Crohn's disease. Clin Exp Immunol 94: 174-181, 1993.

17. Mitsuyama K, Sasaki E, Toyonaga A, et al: Colonic mucosal interleukin-6 in inflammatory bowel disease. Digestion 50: 104-111, 1991.

18. Carey R, Jurickova I, Ballard E, et al: Activation of an IL-6:STAT3-dependent transcriptome in pediatric-onset inflammatory bowel disease. Inflamm Bowel Dis 14: 446-457, 2008.

19. Ishiguro Y: Mucosal proinflammatory cytokine production correlates with endoscopic activity of ulcerative colitis. J Gastroenterol 34: 66-74, 1999.

20. ter Steege JC, van de Ven MW, Forget PP, et al: The role of endogenous IFN-gamma, TNF-alpha and IL-10 in LPS-induced nitric oxide release in a mouse model. Cytokine 10: 115-123, 1998.

21. Lawrence T, Willoughby DA and Gilroy DW: Anti-inflammatory lipid mediators and insights into the resolution of inflammation. Nat Rev Immunol 2: 787-795, 2002.

22. Turini ME and DuBois RN: Cyclooxygenase-2: a therapeutic target. Annu Rev Med 53: 35-57, 2002.

23. Moncada S: Nitric oxide: discovery and impact on clinical medicine. J R Soc Med 92: 164-169, 1999.

24. Mudter J, Weigmann B, Bartsch B, et al: Activation pattern of signal transducers and activators of transcription (STAT) factors in inflammatory bowel diseases. Am J Gastroenterol 100: 64-72, 2005.

25. Musso A, Dentelli P, Carlino A, et al: Signal transducers and activators of transcription 3 signaling pathway: an essential mediator of inflammatory bowel disease and other forms of intestinal inflammation. Inflamm Bowel Dis 11: 91-98, 2005

26. Kubo M, Hanada T and Yoshimura A: Suppressors of cytokine signaling and immunity. Nat Immunol 4: 1169-1176, 2003.

27. Alexander WS and Hilton DJ: The role of suppressors of cytokine signaling (SOCS) proteins in regulation of the immune response. Annu Rev Immunol 22: 503-529, 2004.

28. Grutkoski PS, Chen Y, Chung CS and Ayala A: Sepsis-induced SOCS-3 expression is immunologically restricted to phagocytes. J Leukoc Biol 74: 916-922, 2003. 
29. Corvinus FM, Orth C, Moriggl R, et al: Persistent STAT3 activation in colon cancer is associated with enhanced cell proliferation and tumor growth. Neoplasia 7: 545-555, 2005.

30. Mitsuyama K, Matsumoto S, Rose-John S, et al: STAT3 activation via interleukin 6 trans-signalling contributes to ileitis in SAMP1/Yit mice. Gut 55: 1263-1269, 2006.

31. Isomoto H, Mott JL, Kobayashi S, et al: Sustained IL-6/STAT-3 signaling in cholangiocarcinoma cells due to SOCS-3 epigenetic silencing. Gastroenterology 132: 384-396, 2007.

32. Wang XY and Tian DL: Etiological and pathological characteristics of ulcerative colitis and TCM differentiation and treatment Beijing Zhong Yi Yao Da Xue Xue Bao 30: 554-559, 2007 (In Chinese).

33. Gong YP, Liu W, Ma GT, et al: Randomized control study of 'Qingchang Suppository' on ulcerative colitis. Shanghai Zhong Yi Yao Da Xue Xue Bao 21: 33-36, 2007 (In Chinese).

34. Fu NL and Huang JY: Progress of clinical research of traditional Chinese medicine for the treatment of ulcerative colitis. J Tradit Chin Med 40: 501-503, 1999 (In Chinese).

35. Li QG: An idea about treatment of ulcerative colitis by TCM methods. Beijing Zhong Yi 23: 149-150, 2004 (In Chinese).

36. Wang $\mathrm{CH}$, Gao WY, Li YF, et al: Study of Fufangkushen colon-release capsule on ulcerative colitis of endo-retention of damp heat type. Xian Dai Zhong Xi Yi Jie He Za Zhi 18: 13-15, 2009 (In Chinese)

37. Chen JT, Ke X, Fu XY, et al: The clinical study of heat-clearing and damp-drying on the treatment of damp-heat ulcerative colitis. Zhongguo Zhong Xi Yi Jie He Xiao Hua Za Zhi 17: 256-257, 2009 (In Chinese).
38. Ke X, Zhou F, Gao Y, Xie B, et al: Qing Hua Chang Yin exerts therapeutic effects against ulcerative colitis through the inhibition of the TLR4/NF- $\kappa B$ pathway. Int J Mol Med 32: 926-930, 2013.

39. Ke X, Chen J, Zhang X, et al: Qing Hua Chang Yin attenuates lipopolysaccharide-induced inflammatory response in human intestinal cells by inhibiting NF- $\mathrm{BB}$ activation. Exp Ther Med 6: 189-193, 2013.

40. Levy DE and Darnell J: Stats: transcriptional control and biological impact. Nat Rev Mol Cell Biol 3: 651-662, 2002.

41. Murray PJ: The JAK-STAT signaling pathway: input and output integration. J Immunol 178: 2623-2629, 2007.

42. Pfitzner E, Kliem S, Baus D and Litterst M: The role of STATs in inflammation and inflammatory diseases. Curr Pharm Des 10: 2839-2850, 2004.

43. Okugawa S, Ota Y, Kitazawa T, et al: Janus kinase 2 is involved in lipopolysaccharide-induced activation of macrophages. Am J Physiol Cell Physiol 285: C399-C408, 2003.

44. Zhu Z, Jin H, Yu P, et al: Mollugin inhibits the inflammatory response in LPS-stimulated RAW264.7 macrophages by blocking the Janus kinase (JAK)-signal transducers and activators of transcription. Biol Pharm Bull 36: 399-406, 2013.

45. Greenhalgh CJ, Miller ME, Hilton DJ, et al: Suppressors of cytokine signaling: Relevance to gastrointestinal function and disease. Gastroenterology 123: 2064-2081, 2002. 